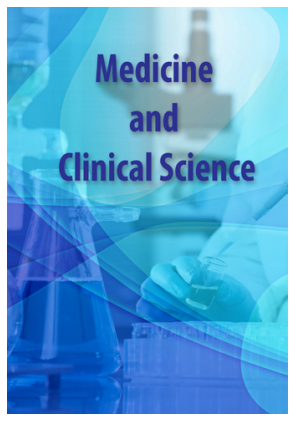

Correspondence

Diane L. Check

7447 Old York Road, Melrose Park, PA 19027 USA

Tel: $215-635-4400$

Fax: 215-635-2304

E-mail:dlk0626@yahoo.com

- Received Date: 22 Nov 2019;

- Accepted Date: 01 Dec 2019;

- Publication Date: 08 Dec 2019.

Copyright

(C) 2019 Science Excel. This is an openaccess article distributed under the term of the Creative Commons Attribution 4.0 International license.

\section{Significant palliative benefits of single agent mifepristone for advanced lung cancer that previously failed standard therapy}

\author{
Diane L Check ${ }^{1 *}$ and Jerome H Check ${ }^{1,2}$ \\ ${ }^{1}$ Cooper Institute for Reproductive Hormonal Disorders, P.C., MT. Laurel, NJ, USA \\ 2Division of Reproductive Endocrinology \& Infertility, Department of Obstetrics \& Gynecology, Cooper Medical School of Rowan \\ University, Camden, NJ, USA
}

\begin{abstract}
A large variety of cancers have been found to make a unique immunomodulatory protein called the progesterone induced blocking factor (PIBF), which is utilized by the fetal-placental unit to escape cellular immune surveillance. The progesterone receptor involved in PIBF production is a membrane receptor. Thus, it is present in cancers, whether the classic nuclear progesterone receptor is present, or not. Controlled studies involving mice with spontaneous cancers, showed increased longevity and palliation when they were gavaged with mifepristone. Similarly, single agent mifepristone promoted extension of life and palliation in several end stage patients with a variety of different cancers. Based on these studies, the FDA granted an investigator initiated 40 patient study entitled "A phase II study of treatment with oral mifepristone as salvage therapy in patients with advanced or metastatic non-small lung cancer who have failed two or more previous chemotherapy regimens". The first case enrolled is a 65 year old male with stage IV non-small cell lung adenocarcinoma with a brain metastasis. He progressed despite 3 rounds of chemotherapy cocktails. Because his tumor was devoid of any markers that would respond to targeted therapy, his oncologist referred him for the single agent oral mifepristone trial (300mg per day). This patient has completed 4 years of therapy. He feels great, despite the fact that over the last 6 months there has been progression of his lung nodules. There has not been any more brain metastases. He has no side effects from the mifepristone. Very rarely a patient with end stage lung cancer will have a complete response. Thus, the main objective of the study is to provide treatment that will provide a decent and extended quality of life with no suffering from the treatment itself. At least in this case, mifepristone therapy seems to meet these criteria.
\end{abstract}

\section{Introduction}

In 2001, a hypothesis was put forth that some malignant tumors could produce an immunomodulatory protein, known as the progesterone induced blocking factor (PIBF), which, similar to pregnant women, where it helps the fetus to escape immune surveillance, could be also utilized by cancer cells to similarly avoid immune destruction [1]. Support for this hypothesis was provided by the demonstration that, not only did certain cancer cell lines produce a very high level of messenger ribonucleic acid (mRNA) for the PIBF protein, but that adding progesterone to the media up-regulated the PIBF protein, whereas, adding the progesterone receptor modulator, mifepristone, resulted in down-regulation of this protein $[2,3]$.

To determine if mifepristone could be clinically useful as an anticancer drug, several controlled trials were conducted in spontaneous murine cancer models. Efficacy of the drug, versus placebo, was found in mice with leukemia, testicular cancer, prostate cancer and lung cancer[4-6].

Mifepristone, a drug already approved on the pharmaceutical market as an anti-abortifacient, had a restricted use, to satisfy strong anti-abortion groups, to be used only by licensed abortionists. This precluded a physician from prescribing it offlabel, without special permission from the Food and Drug Administration (FDA) in the United States. Based on cancer cell line data and animal data, the FDA granted a compassionate use investigational new drug (IND) approval, on a case by case basis, for patients with advanced cancer, when they had failed standard treatments, and there did not appear to be any further treatment options. This led to significant improvement in length and quality of life in anecdotal cases with several different types of advanced cancer [7-10].

Based on experimental data suggesting a positive benefit of mifepristone involving cancer cell lines, controlled murine cancer studies, and anecdotal cases of patients with advanced cancer providing palliative benefits, the FDA approved an investigator initiated study for 40 patients entitled "A phase II study of treatment with oral mifepristone as salvage therapy in patients with advanced or metastatic non-small lung cancer who have failed two or more previous chemotherapy regimens". We report, here, the outcome to date of the first case registered to this investigator initiated study.
Citation: Check DL. Significant palliative benefits of single agent mifepristone for advanced lung cancer that previously failed standard therapy. Med Clin Sci. 2019;1(2):1-5. 


\section{Case Report}

In December, 2015, a 68 year old man with a 50 pack year history of smoking, presented to our office for consideration for enrollment in our FDA granted investigator initiated study. He had been diagnosed in September 2013, while undergoing pre-testing for a urinary bladder resection, for a papillary urothelial carcinoma. A pre-surgery chest $\mathrm{x}$-ray showed lung lesions, and a subsequent core biopsy of a right lung nodule confirmed adenocarcinoma.

Immunohistochemical studies performed at that time were positive for cytokeratin (CK)7, Napsin A, thyroid transcription factor 1 (TTF-1), and negative for CK 5/6, CK 20, and tumor protein 63 (P63). Staging computerized tomography (CT) findings showed bilateral lung lesions, including a $3.3 \times 2.7$ left upper lobe mass (LUL), 11 x 10mm solid nodule in right lower lobe (RLL), subcentimeter, part solid, groundglass LUL and a $1.6 \mathrm{~cm}$ right lower paratracheal node. Besides bladder and lung cancer, he also was diagnosed with chronic obstructive lung disease.

After his bladder resection, he started treatment of his lung cancer. He received 6 cycles of chemotherapy (chemo), carboplatin/avastin/ docetal, from October, 2013 to February 2014. Post-chemo CT, April 2014, showed a partial response, with the LUL mass decreasing to 1.3 $\mathrm{x} 1.1 \mathrm{~cm}$. The other nodules were all unchanged, compared to baseline scan. He then received 6 cycles of pemetrexed, March, 2014 to June, 2014. Follow up position emission tomography (PET)/CT scan in July, showed continued response, with resolution of the right lower paratracheal node, and the other lesions were non-fluoro-deoxyglucose (FDG) avid and unchanged from size, from previous scan. No other treatment was given and his next CT scan in November, 2014 remained stable. Unfortunately, in February, 2015, his PET/CT scan, indicated recurrence. There was FDG uptake in the lower right paratracheal and right lower hilar lymph nodes. On CT attenuation, these measured $1.2 \mathrm{x}$ $1.1 \mathrm{~cm}$ and $1.4 \times 1.0 \mathrm{~cm}$, respectively. There was also a $1.8 \times 1.3 \mathrm{~cm}$ mildly avid pulmonary nodule.

He then received 6 cycles of gemcitabine/carboplatin, from April, 2015 to September, 2015. Follow-up PET/CT showed stable right hilar node, and interval decrease is size of RLL nodule. The previous hilar node improved to non avid. In October he had a seizure like episode and magnetic resonance imaging (MRI) of the brain indicated a $1 \mathrm{~cm}$ right frontal lobe lesion. He received sterostatic radiosurgery therapy, with 2500 centigray (cGy), in 5 fractions, completed in November, 2015.
Treatment plan CT at this time, showed new avid LUL nodules and the original biopsy was sent for programmed death-ligand 1 (PD-L1) testing and was found to be negative. Clinically, he was having shortness of breath, fatigue and a mild anemia. After reviewing treatment options with his oncologist, he decided to come for screening for our single agent mifepristone study, and didn't have the follow-up brain MRI.

After signing the Chesapeake institutional review board (IRB) approved consent form and having the necessary pre-testing, including the Quality of Life questionnaires (QLQ), European Organization for Research and Treatment of Cancer (EORTC) QLQ-30 and EORTC QLQ-lung cancer (LC)13, he started on daily $300 \mathrm{mg}$ oral mifepristone (Korlym), provided by Corcept Inc., January 8th, 2016. His baseline PET/CT scan from December, 2015 indicated multiple new FDG avid LUL nodules, largest measuring $2.3 \times 1.2 \mathrm{~cm}$ on CT attenuation, RUL $1.5 \mathrm{~cm}$ nodule and a stable hilar node. On baseline physical exam and review of systems, he said he was having a little shortness of breath, especially going up stairs, and little less energy, but was able continue working in a band, although, he had to sit towards the end of a set and was a little hoarse. We considered him an ECOG 1, although a Karnofsky Performance Status (KPS) of 90 would give a better picture of him at the time. His lungs were clear at the visit, but his visit to the oncologist 2 weeks before, showed that he had some decreased breath sounds in right lower lung. All other systems were normal.

In his follow-up visit 1 month later, he stated that he had more energy, he was no longer hoarse like he was before, and he could now walk up stairs with no shortness of breath. He had just seen his oncologist who said his lungs sounded clear. His hemoglobin also went up a gram. He was assessed as an ECOG 0, and now would be considered as having a KPS score of 100 . He had no adverse events to the medication.

His follow-up CT at the end of 2 months showed a mild increase in his LUL lesion to $2.9 \times 3 \mathrm{~cm}$ and a slight increase in his RLL nodule to 1.7 $\mathrm{x} 1.0$, now avid.

At 5 months he had a slight decrease in size of RUL and the RLL appeared less nodular. His brain CT showed even more decrease in the previous radiated lesion, now measuring $0.8 \mathrm{~cm}$. Clinically, he said he never felt better.

\begin{tabular}{|c|c|}
\hline Comparison: & CT thorax dated March 21, 2019 \\
\hline \multirow[t]{2}{*}{ Findings: } & $\begin{array}{l}\text { Central airways/lung parenchyma/pleura: Increased size necrotic right lower lobe mass, measuring } 7.9 \text { x } 6.3 \times 5.1 \mathrm{~cm} \\
\text { (previously } 7.3 \times 6.1 \times 4.9 \mathrm{~cm} \text { ), with extension to the right hilum and increased postobstructive atelectasis within the } \\
\text { right lower lobe. Slightly increased } 1.7 \mathrm{~cm} \text { nodular branching opacity within the superior segment of the right lower lobe. } \\
\text { Increased size } 2.0 \mathrm{~cm} \text { right lower lobe subpleural nodule (series } 4 \text {, image } 144 \text { ), previously } 0.9 \mathrm{~cm} \text {. } \\
\text { Multiple additional bilateral upper lobe predominant pulmonary nodules, some of which contain cavitation, are overall } \\
\text { unchanged, for example an } 8 \mathrm{~mm} \text { right upper lobe cavitary nodule (series } 4 \text {, image } 52 \text { ). Stable } 1.4 \mathrm{~cm} \text { linearly oriented } \\
\text { left upper lobe nodular opacity (series } 4 \text {, image } 89 \text { ). } \\
\text { Mediastinum/great vessels: Heart size is within normal limits. Multiple subcentimeter and mildly enlarged bilateral hilar } \\
\text { and mediastinal lymph nodes have overall slightly increased in size. For example: } \\
\text { - Slightly increased conglomerate subaortic adenopathy measures } 4.3 \times 2.4 \mathrm{~cm} \text { (previously } 3.1 \times 1.2 \mathrm{~cm} \text { ) } \\
\text { - Slightly increased } 0.8 \mathrm{~cm} \text { prevascular adenopathy (previously } 0.3 \mathrm{~cm} \text { ) } \\
\text { - Stable right hilar adenopahy measures } 2.4 \text { x } 1.7 \mathrm{~cm} \\
\text { Osseous structures: Degenerative changes of the spine. No suspicious osseous lesion. }\end{array}$ \\
\hline & $\begin{array}{l}\text { 1. Since March 21, 2019, slightly increased } 7.9 \mathrm{~cm} \text { necrotic right lower lobe mass, with increased right lower lobe post- } \\
\text { obstructive atelectasis. } \\
\text { 2. Multiple additional bilateral pulmonary nodules are overall stable to slightly increased in size. } \\
\text { 3. Multiple subcentimeter and mildly enlarged mediastinal and hilar lymph nodes have overall slightly increased in size. }\end{array}$ \\
\hline
\end{tabular}

Table 1. CT Chest, July 10, 2019 


\begin{tabular}{|c|c|}
\hline Comparison: & CT thorax dated March 21, 2019 \\
\hline Findings: & $\begin{array}{l}\text { Tubes/Lines: None. } \\
\text { Lungs, pleura, and airways: Increased size of the heterogeneous, partially necrotic right lower lobe mass measuring } 8.2 \\
\text { x } 7.7 \times 7.0 \mathrm{~cm} \text {, previously } 7.9 \times 6.3 \times 5.1 \mathrm{~cm} \text {. Stable extension into the right hilum with encasement and compression of } \\
\text { the right lower lobe pulmonary artery and bronchus. Increased size of a right lower lobe satellite nodule measuring } 2.4 \\
\mathrm{~cm} \text {, previously } 2 \mathrm{~cm} \text { (series } 4 \text {, image } 146 \text { ). Unchanged nodular branching opacities in the superior segment of right lower } \\
\text { lobe, contiguous with the mass measuring up to } 1.8 \mathrm{~cm} \text {. Additional slightly increased bilateral pulmonary nodules. For } \\
\text { example: } \\
\text { - Right upper lobe } 1.4 \mathrm{~cm} \text { nodule, previously } 1.1 \mathrm{~cm} \text { (series } 4 \text {, image } 91 \text { ) } \\
\text { - Left upper lobe tubular nodule measuring up to } 1.8 \mathrm{~cm} \text {, previously } 1.3 \mathrm{~cm} \\
\text { Unchanged few bilateral upper lobe groundglass nodules (for example, series } 4 \text {, image } 31 \text { ). } \\
\text { Mediastinum/lymph nodes: Increased mediastinal and hilar lymphadenopathy. For example: } \\
\text { - Prevascular } 1.3 \times 1.1 \mathrm{~cm} \text { lymph node, previously subcentimeter } \\
\text { - Subaortic } 2.2 \times 1.5 \mathrm{~cm} \text { lymph node, previously } 1.9 \times 1 \mathrm{~cm} \\
\text { - Right hilar } 1.9 \times 1.5 \mathrm{~cm} \text { lymph node, previously } 1.8 \text { x } 1.3 \mathrm{~cm} \\
\text { - Left hilar } 2.5 \times 1.2 \mathrm{~cm} \text { lymph node, previously } 1.5 \times 1.5 \mathrm{~cm} \\
\text { Partially imaged left supraclavicular lymphadenopathy. } \\
\text { Heart/Great vessels: Normal heart size. No pericardial effusion. Stable atherosclerotic vascular calcifications. } \\
\text { Bones/Soft tissues: No suspicious osseous lesions. Stable degenerative changes of the thoracic spine. } \\
\text { Visualized upper abdomen: Unremarkable. }\end{array}$ \\
\hline Impression & $\begin{array}{l}\text { 1. Since July 10, 2019, increased size of the partially necrotic right lower lobe mass measuring } 8.2 \times 7.7 \times 7.0 \mathrm{~cm} \text {, previ- } \\
\text { ously } 7.9 \times 6.3 \times 5.1 \mathrm{~cm} \text {; additional bilateral solid pulmonary nodules have all slightly increased in size. } \\
\text { 2. Increased mediastinal and hilar lymphadenopathy. }\end{array}$ \\
\hline
\end{tabular}

Table 2. CT Chest October 30, 2019

Unfortunately, his next CT scan showed the RLL increasing in size and FDG uptake (72\%) and new pathological uptake in the right hilar node. There was a decreased FDG uptake in the LUL nodule. He still felt great and had not side effects to the medication.

Throughout his fifty-one 28 day cycles (about 4 years), of single agent mifepristone therapy, some lesions increased while others decreased, but he remained "feeling great". Most of his monthly 43 questions quality of life evaluations were answered "not at all".

Despite evidence of progression in his lung CT scans, performed every 2-3 months, he continues to feel great and remains ECOG-0. His last 2 CT's of the chest are seen in Table $1(7 / 10 / 19)$ and Table $2(10 / 30 / 19)$. Since the primary objectives of this study are quality of life and survival with disease progression as a secondary objective, he continues the single agent mifepristone treatment.

The decision to remain on single agent mifepristone was made based on the fact that there are no other treatment options for this patient. Although he is showing radiographic progression, clinically, he remains significantly improved. Furthermore, it has been our experience that stopping the drug results in rapid progression of the cancer.

The hope is that the drug can continue to provide slowing of cancer advancement and to give him even more months of a high quality of life.

Should he clinically deteriorate, we might consider determining if continuing mifepristone would allow him to respond once again to a previous chemotherapy cocktail to which he initially demonstrated response, but became resistant. This would remove him from the study, but hopefully Corcept, Inc. may still provide the 300mg dosage of mifepristone gratis, but if not, he could be switched to the $200 \mathrm{mg}$ tablet that would then require a compassionate use IND, but would be much less expensive than the $300 \mathrm{mg}$ tablet.

\section{Discussion}

5A case report, such as the one presented here, can help provide very convincing evidence that, in some instances, mifepristone can significantly increase both length and quality of life, in at least one patient (which hopefully implies probably more), with stage IV advanced nonsmall cell lung cancer, even with brain metastases, despite progressing with extensive standard chemotherapy regimens. Though palliative radiotherapy may have been responsible for stopping the progression of the one brain metastatic lesion, since expectation would be to see more brain metastases, it seems likely that in this case, the drug crosses the blood brain barrier, supporting a case of a glioblastoma multiforme, stage IV, in which mifepristone therapy seemed to provide palliation [9].

One may think that this case probably represents the longest survival with good quality of life of any case with lung cancer treated with single agent mifepristone, in view of completing 50 twenty-eight day cycles of mifepristone. Actually, there was a previous report of a woman with a very rapid progressive presentation of lung cancer who also had the syndrome of inappropriate anti-diuretic hormones (serum sodium $118 \mathrm{mmol} / \mathrm{L}$ ). She had complete remission of all her lung nodules, and restoration of her serum sodium to normal, with single agent mifepristone therapy. She lived more than 5 years, dying not from her lung cancer, but an acute myocardial infarction [10].

The second case from this investigator initiated study of single agent mifepristone for non-small cell lung cancer, has been previously published. She had also shown marked clinical improvement of her stage IV non-small cell lung cancer with single agent treatment with $300 \mathrm{mg}$ oral mifepristone, which attained stable disease. Unfortunately her death $1 \frac{1 / 2}{2}$ years after initiating mifepristone therapy was not from the advancement of her lung cancer, but end stage chronic obstructive lung disease [11]. The important difference from the first case, 
presented here, is that this patient progressed not only despite several courses of standard chemotherapy, but also progressed with the checkpoint inhibitor, nivolumab, because she was positive for the PD-L1 marker [11].

The present case, in his 45 months (51 cycles) of treatment, has shown stable disease. The tumor response to mifepristone in published case reports of palliative benefits, have, in a minority of cases, shown complete remission, but the majority have demonstrated partial remission, or stable disease [7-11]. That is why the primary objective for the larger FDA approved investigator initiated study are quality and length of life compared to expected, with disease progression as a secondary endpoint.

Certain cancers have the presence of receptors in the nucleus for sex steroids, e.g., estrogen and progesterone. Indeed, one of the main treatment modalities for preventing recurrence, or spread of breast cancer, positive for the classic nuclear estrogen receptor, e.g., breast and endometrial cancer, is to suppress or block the effects of estrogen. The thought behind this treatment is that the hormones interacting with its nuclear receptor, in some way, enables the malignant tumor to grow.

Similar thoughts extended to tumors that were positive for the classic nuclear progesterone receptor. Mifepristone therapy had been previously demonstrated to cause a complete or partial remission, or stable disease, in some women with either tamoxifen resistant or untreated metastatic breast cancer that were positive for the nuclear progesterone receptor [12-14]. However, it was not realized that even if there was some response some tumors could still be growing, and the continued use of mifepristone could still provide a reasonable extension of a good quality life. Indeed, several times the patient in this reported study showed evidence of disease progression, but in contrast to other therapies, where once progression is demonstrated the spread of the cancer accelerates, this does not appear to be the case with mifepristone therapy. In fact, in the authors' experience cessation of the drug may lead to rapid acceleration of disease advancement [7-11,15,16]. Not evaluating the proper objectives to mifepristone therapy, has led to opposite opinions of its efficacy in another cancer (ovarian) associated with the classic nuclear progesterone receptor, in two published studies by the same lead author $[17,18]$.

Except for a small minority of cases, lung cancer is not associated with the classic nuclear progesterone receptor. Thus, one might ponder as to what mechanism is involved in allowing the progesterone receptor modulator, mifepristone, (generally an antagonist but not against all progesterone receptors) to be so effective for a variety of cancers, besides lung cancer, that are not associated with the presence of the classic nuclear progesterone receptor [7-11]. The hypothetical model favored by our group, and some others, is that a membrane progesterone receptor is involved in the production of the immunosuppressive intracytoplasmic PIBF protein, which is produced by the cancer cells, similar to embryonic, trophoblast, and mesenchymal cells of the fetalplacental unit, which when released into the microenvironment inhibits the cellular immune attack against the fetus or cancer cells [15,16,1921]. Mifepristone, in turn, by blocking the membrane progesterone receptor, inhibits PIBF production, which allows cellular immune destruction of the cancer cells $[15,16,19-, 21]$.

As mentioned, very convincing case reports may allow conclusions that mifepristone can, in some instances, provide an extended improved quality of life in patients with advanced and metastatic non-small cell lung cancer. Though mifepristone did not show benefit for unresectable meningiomas (possibly these become tumors are too slow growing), the large number of cases of meningiomas treated with mifepristone, clearly demonstrated that daily mifepristone is extremely well tolerated with no significant health risks [22]. We eagerly await the conclusion of the 40 patient study to determine what percentage of patients with stage IIIB or IV non-small cell lung cancer, now recalcitrant to standard therapy, may receive palliative benefits from single agent oral mifepristone therapy.
In evaluating the efficacy of mifepristone, the authors think that the main determinant of efficacy should be longevity and quality of life, rather than progressive disease. As of late, this patient is starting to show some growth of tumors. However, he feels "great", and based on other cases, once the lesions start growing this is not a harbinger for rapid progression. Instead, the drug will hopefully keep his lesions from growing more rapidly, consistent with extension of a good quality of life. For this reason, we are keeping him on the study, rather than assume it is no longer working, and try something else, e.g., a checkpoint inhibitor, even though he is negative for the PD-L1 marker. If progressive disease was our primary endpoint, his mifepristone therapy may have been stopped 2 years before. To reiterate, it has been our experience that if one stops the mifepristone, the cancer will spread rapidly, and his quality of life will diminish.

The last 2 chest CT scans are presented in detail to demonstrate that radiographic progression of the cancer is still consistent with a high quality of life. He still works in a band, and he is able to stand the entire segment during long engagements. He has not had the least bit of shortness of breath, or feels weakness.

By not placing him again on chemotherapy that failed before, or placing him on check-point inhibitors without any PD-L1 markers, we are keeping him free of side effects of these medications. Too often the patient's quality of life is affected by side effects of the cancer treatment, and life's functions are interrupted for hospital admission for intravenous infusions of chemotherapy and side effects of these medications.

If he becomes symptomatic from tumor growth reaching a level that interferes with his quality of life, then the oncologist on this team will help us determine what type of course of action would be best for this patient to relieve symptoms, while maintaining mifepristone to prevent rapid spread, not just to the lungs, but outside the thorax, e.g., the brain. It would be interesting if the use of mifepristone could enable him to once again respond to a chemotherapy cocktail to which he initially responded, but to which he subsequently became resistant. It should be mentioned that radiologic exams of his brain by MRI have all been negative during his 4 years of treatment with mifepristone.

In summary, at least for the first case registered for the investigator initiated evaluation of treatment, this drug has fulfilled the objectives of treatment - to significantly extend a good quality of life beyond expectation with this well tolerated safe treatment.

This case illustrates one more important point. Future studies evaluating mifepristone, or other progesterone receptor antagonists or modulators for lung cancer, or other cancers, should use as their primary determination of efficacy, improved length and quality of life, as opposed to progressive disease.

\section{Conflicts of interest}

The authors hold no patents or patent pending for progesterone receptor modulators, e.g., mifepristone or any drug for that matter.

The authors received no financial compensation from any pharmaceutical company.

\section{Acknowledgement}

We thank Corcept, Inc. for providing the $300 \mathrm{mg}$ mifepristone to this patient at no charge.

We thank Dr. Mohamoud Aly for the kind referral of this patient and his oncology care.

\section{References}

1. Check Check JH, Nazari P, Goldberg J, et al. A model for potential tumor immunotherapy based on knowledge of immune mechanisms responsible for spontaneous abortion. Med Hypoth 2001;57:337-343.

2. Srivastava MD, Thomas A, Srivastava Bl, et al. Expression and modulation of progesterone induced blocking factor (PIBF) and innate 
immune factors in human leukemia cell lines by progesterone and mifepristone. Leuk Lymphoma 2007;48:1610-1617.

3. Check JH, Dix E, Sansoucie L. Support for the hypothesis that successful immunotherapy of various cancers can be achieved by inhibiting a progesterone associated immunomodulatory protein. Med Hypoth 2009;72:87-90.

4. Check JH, Sansoucie L, Chern J, et al. Mifepristone treatment improves length and quality of survival of mice with spontaneous leukemia. Anticancer Res 2009;29(8):2977-2980.

5. Check JH, Dix E, Wilson C, et al. Progesterone receptor antagonist therapy has therapeutic potential even in cancer restricted to males as evidenced from murine testicular and prostate cancer studies. Anticancer Res 2010;30:4921-4924.

6. Check JH, Sansoucie L, Chern J, et al. Mifepristone treatment improves length and quality of survival of mice with spontaneous lung cancer. Anticancer Res 2010;30:119-122.

7. Check JH, Dix E, Sansoucie L, et al. Mifepristone may halt progression of extensively metastatic human adenocarcinoma of the colon - case report. Anticancer Res 2009 May;29(5):1611-1613.

8. Check JH, Dix E, Cohen R, et al. Efficacy of the progesterone receptor antagonist mifepristone for palliative therapy of patients with a variety of advanced cancer types. Anticancer Res 2010;30:623-628.

9. Check $\mathrm{JH}$, Wilson $\mathrm{C}$, Cohen $\mathrm{R}$, et al. Evidence that mifepristone, a progesterone receptor antagonist can cross the blood brain barrier and provide palliative benefits for glioblastoma multiforme grade IV. Anticancer Res 2014;34:2385-2388.

10. Check JH, Check D, Wilson C, et al. Long-term high-quality survival with single-agent mifepristone treatment despite advanced cancer. Anticancer Res 2016;36:6511-6513.

11. Check JH, Check D, Poretta T. Mifepristone extends both length and quality of life in a patient with advanced non-small cell lung cancer that has progressed despite chemotherapy and a check-point inhibitor. Anticancer Res 2019;39:1923-1926.

12. Ramieu G, Maudelonde T, Ulmann A, et al. The antiprogestin RU486 in advanced breast cancer: preliminary clinical trial. Bulletin Du Cancer 1987;74:455-461.

13. Klijin JG, Setyono-Han B, Foekens JA. Progesterone antagonists and progesterone receptor modulators in the treatment of breast cancer. Steroids 2000;65:825-830.

14. Perrault D, Eisenhauer EA, Pritchaard Kl, et al. Phase II study of the progesterone antagonist mifepristone in patients with untreated metastatic breast carcinoma: a National Cancer Institute of Canada Clinical Trials Group Study. J Clin Oncol 1996;14:2709-2712.

15. Check $\mathrm{JH}$, Cohen R. The role of progesterone and the progesterone receptor in human reproduction and cancer. Exp Rev Endocrinol Metab 2013;8:469-484.

16. Check JH. The role of progesterone and the progesterone receptor in cancer. Expert Review Endo Metab 2017;12:187-197.

17. Rocereto TF, Saul HM, Aikins JA Jr, et al. Phase II study of mifepristone (RU486) in refractory ovarian cancer. Gynecol Oncol 2000;77:429-432.

18. Rocereto TF, Brady WE, Shahin MS, et al. A phase II evaluation of mifepristone in the treatment of recurrent or persistent epithelial ovarian, fallopian or primary peritoneal cancer: A gynecologic oncology group study. Gynecol Oncol 2010;116:332-334.

19. Cohen RA, Check JH, Dougherty MP. Evidence that exposure to progesterone alone is a sufficient stimulus to cause a precipitous rise in the immunomodulatory protein the progesterone induced blocking factor (PIBF). J Assist Reprod Genet 2016;33:221-229.

20. Check JH, Check D. Therapy aimed to suppress the production of the immunosuppressive protein progesterone induced blocking factor (PIBF) may provide palliation and/or increased longevity for patients with a variety of different advanced cancers - a review. Anticancer Res 2019;39(7):3365-3372.

21. Szekeres-Bartho J, Polgar B. PIBF: the double edged sword. Pregnancy and tumor. Am J Reprod Immunol 2010;64:77-86.

22. Ji Y, Rankin C, Grunberg S, et al. Double-blind phase III randomized trial of the antiprogestin agent mifepristone in the treatment of unresectable meningioma: SWOG S900S. J Clin Oncol 2015;33:40934098. 